\title{
INFLUÊNCIAS NÃO LINEARES DA INDÚSTRIA NO DESEMPENHO DA FIRMA
}

\author{
The non-linear industry effects on firm performance \\ Influencias no lineales de la industria en el desempeño de la empresa
}

\begin{abstract}
RESUMO
Esta pesquisa tem por objetivo investigar o efeito do ambiente da indústria no desempenho da firma, por meio de um modelo de regressão múltipla não linear. As amostras por período variam de 178 (2005) a 252 (2012) firmas brasileiras de capital aberto, ativas no período de 2005 a 2012. A análise por decomposição da variância permitiu a identificação de tendência de incremento da influência da rivalidade no desempenho da firma, ao passo que a relação quadrática aponta para um ponto ótimo do efeito da rivalidade na indústria que, caso ultrapassado, poderá provocar efeito inverso na lucratividade. Tais resultados estão alinhados com a perspectiva de que, quanto maior a rivalidade, mensurada por meio da receita dos concorrentes, maior a possibilidade de obtenção de receitas originadas da venda de produtos, até o ponto em que a intensidade da rivalidade passa a dificultar o acesso a recursos oriundos das vendas.
\end{abstract}

PALAVRAS-CHAVE | Desempenho, vantagem competitiva, lucratividade, ambiente da indústria, regressão múltipla não-linear.

\section{ABSTRACT}

This paper aims to investigate the effects of the industry environment on firm's performance, by using a non-linear regression model. The samples used range from 178 (2005) to 252 (2012) publicly traded Brazilian firms, active in the period from 2005 to 2012. The analysis, which uses the variance decomposition method, allows one to identify a tendency for the influence of rivalry to increase a firm's performance. The quadratic relationship identified here points to an equilibrium point of the industry environment effect that, if exceeded, may cause a reverse effect on profitability. These results are in line with the view that as rivalry increases, as measured in terms of rivals' performance, so the possibility of obtaining a better sales performance also increases, until the point where the level of rivalry intensity becomes a barrier to increasing sales any further.

KEYWORDS / Performance, competitive advantage, profitability, industry environment, non-linear multiple regression.

\section{RESUMEN}

Este estudio tiene como objetivo investigar el efecto del ambiente de la industria en el desempeño de la empresa, por medio de un modelo de regresión múltiple no lineal. Las muestras por período varían de 178 (2005) a 252 (2012) empresas brasileñas de capital abierto, activas en el período de 2005 a 2012. El análisis por descomposición de la varianza permitió la identificación de tendencia de incremento de la influencia de la rivalidad en el desempeño de la empresa, mientras que la relación cuadrática apunta hacia un punto excelente del efecto de la rivalidad en la industria que, en caso de ser superado, podrá provocar efecto inverso en la lucratividad. Tales resultados están alineados con la perspectiva de que, cuanto mayor la rivalidad, medida por medio de los ingresos de los competidores, mayor la posibilidad de obtención de ingresos originados de la venta de productos, hasta el punto en que la intensidad de la rivalidad comienza a dificultar el acceso a recursos oriundos de las ventas.

PALABRAS CLAVE I Desempeño, ventaja competitiva, lucratividad, ambiente de la industria, regresión múltiple no lineal. 


\section{INTRODUÇÃO}

As decisões estratégicas são definidas em confluência com a área de atuação das firmas, com os padrões de desempenho esperados para si e de modo a equilibrar as pressões oriundas das restrições econômicas; ou seja, a firma opera em um ambiente particular com objetivos de desempenho definidos (Child, 1972).

As relações entre fatores ligados à indústria e ao desempenho da firma têm sido abordadas por vários autores (e. g. Child, 1974; Hatten \& Schendel, 1977; Mauri \& Michaels, 1998; Prescott, 1986; Roquebert, Phillips, \& Westfall, 1996; Rumelt, 1991; Schmalensee, 1985; Tirole, 1988), entre os quais há aqueles que consideram os efeitos da indústria de uma forma mais ampla (Bamiatzi \& Hall, 2009; Hawawini, Subramanian, \& Verdin, 2003; Kim \& Reinschmidt, 2012) e os de suas características, em termos de munificência, instabilidade e complexidade (Keats \& Hitt, 1988), de concentração (Griffiths, Jensen, \& Webster, 2011; Sharma, 2011) e rivalidade (Cool \& Dierickx, 1993; Cool, Dierickx, \& Jemison, 1989; Mas-Ruiz \& Ruiz-Moreno, 2011), que, em sua maioria, abordam tais relações sob uma perspectiva linear.

Em alinhamento com as perspectivas dos autores e com as proposições de Tirole (1988) acerca da natureza não linear das relações entre contexto competitivo e desempenho da firma, tendo em vista a competição baseada em preços, esta pesquisa tem como objetivo contribuir para essa temática por meio da análise da variação, ao longo do tempo e sob uma perspectiva não linear, da influência da indústria no desempenho da firma, no contexto brasileiro. O contexto competitivo da indústria foi caracterizado por meio da rivalidade da indústria e o desempenho da firma, por meio da lucratividade.

Segundo Cameron e Whetten (1983, como citado em Matitz e Bulgacov, 2011, (p. 582)), os esforços das pesquisas direcionadas ao estudo do desempenho impactam diretamente os modelos de efetividade da firma, que apresentam implicações: “(a) teóricas, pois se encontra no centro de todos os modelos organizacionais; (b) empíricas, pois é a variável dependente final em pesquisa organizacional e; (c) práticas, pois há necessidade de avaliar os resultados obtidos pelas organizações”.

Como contribuição para as pesquisas acerca dessa temática, este artigo investiga a influência do ambiente da indústria no desempenho de firmas brasileiras, por meio da proposição de um modelo de regressão múltipla não linear, aplicado a uma amostra que varia de 178 (2005) a 252 (2012) firmas brasileiras de capital aberto ativas no período entre 2005 e 2012, que apresentaram informações contábeis completas na base Economática ${ }^{\circledR}$, publicaram informações trimestrais na base da Comissão de Valores Mobiliários e tiveram ações negociadas em bolsa nesses mesmos anos. 0 estudo de tal período foi realizado em função da crise econômica ocorrida em 2008, nos Estados Unidos, englobando tanto períodos que a precederam (de 2005 a 2007) quanto que a sucederam (de 2009 a 2012), assim como o próprio ano de 2008, cujos reflexos, com diferentes intensidades, alcançaram âmbito mundial. Os resultados da estimação do modelo proposto confirmam a existência de uma relação não linear entre a rivalidade na indústria e o desempenho da firma, com aumento da sua capacidade explicativa ao longo do tempo.

A opção pela inclusão somente de firmas brasileiras na amostra deu-se em função da emergência econômica do País em termos mundiais, durante o período considerado para estudo, e pelo seu alinhamento com outras pesquisas que abordaram aspectos econômicos, financeiros e competitivos de tais firmas, a exemplo da pesquisa desenvolvida por Pimentel e Aguiar (2016).

O artigo está estruturado de modo a apresentar, inicialmente, discussões conceituais acerca das relações entre lucratividade, desempenho e vantagem competitiva, passando pelo papel dos condicionantes ambientais na determinação do desempenho das firmas e chegando à proposição das hipóteses de pesquisa que buscam abordar as lacunas deixadas por artigos empíricos desenvolvidos sob a mesma temática. A seguir, são apresentados os aspectos metodológicos da pesquisa, englobando a operacionalização e mensuração das variáveis, a coleta de dados e a composição da amostra. Logo em seguida, são apresentados e discutidos os resultados da análise de dados, para depois serem tecidas as considerações e conclusões.

\section{LUCRATIVIDADE, DESEMPENHO E VANTAGEM COMPETITIVA}

Lewin e Minton (1986, como citado em Matitz e Bulgacov, 2011) atentam para o fato de que as pesquisas empíricas ainda não foram capazes de desenvolver uma teoria universal de efetividade organizacional. Ainda assim, deve-se ter claro o longo caminho percorrido durante as últimas décadas de pesquisas em estratégia, e, mais especificamente, de pesquisas sobre a influência do ambiente e seu impacto no desempenho organizacional.

Segundo Richard, Devinney, Yip, e Johnson (2009), “a competição de mercado por clientes, insumos e pelo capital torna o desempenho organizacional essencial para a sobrevivência e para o sucesso da empresa moderna" (p. 719). A fim de viabilizar esses objetivos, Simerly e Li (2000, p. 37) mencionam que a gestão estratégica enxerga a firma como uma organização de múltiplos personagens, interesses e objetivos, sendo impossível maximizar o retorno de todos ou alcançar todos os objetivos. 0 
raciocínio dos autores culmina no fato de que a estratégia se preocupa com a sobrevivência da firma dentro do seu ambiente e que isso requer modelos mais complexos para a sua gestão.

Richard et al. (2009), inspirados em Venkatraman e Ramanujam (1986), afirmam que "o desempenho é um tipo de indicador da eficácia, com vantagens e desvantagens" (p. 722). Assim, eles diferenciam desempenho e eficácia com a seguinte definição (Richard et al., 2009):

- Desempenho organizacional: abrange três áreas específicas de resultados: (a) a lucratividade; (b) o desempenho do mercado de produtos (vendas, participação de mercado etc.); e (c) o retorno dos acionistas.

- Eficácia organizacional: é mais ampla e captura o desempenho organizacional além da diversidade de resultados do desempenho interno. Normalmente está associada às operações mais eficientes ou eficazes, além de incluir outras medidas externas, como é o caso da responsabilidade social corporativa.

Conforme coloca Schilke (2014), a posição de uma firma em seu ambiente competitivo permite identificar a ocorrência de vantagem competitiva, quando seu desempenho está situado em patamares superiores aos de seus competidores, confirmando a proposição de Powell (2001) de que o desempenho superior está diretamente ligado ao conceito de vantagem competitiva. Brito e Brito (2012) afirmam que, quando a maioria dos estudos empíricos se direciona apenas a modelar métricas de desempenho, deixa à margem o alcance e a manutenção de um patamar superior de desempenho, diante da indústria, que deveria ser o cerne de todos esses estudos.

A maioria dos estudos empíricos infere a existência de vantagem competitiva em casos de desempenho superior (Powell, 2001). Ainda de acordo com Brito e Brito (2012), a análise da lucratividade abrange o domínio da apropriação de todo o valor da firma definido pelo valor de troca (preço e custo), desconsiderando outros resultados, como o desenvolvimento de recursos e capacidades com seus parceiros comerciais. Ao tratar do conceito de vantagem competitiva, Brito e Brito (2012) vão além de Powell (2001), pois a definem não apenas como desempenho superior, mas como criação de valor superior em relação aos concorrentes, estando alocada como um antecedente do desempenho, o que possibilita que a criação de valor superior se manifeste também no desempenho financeiro da firma.

Brito e Brito (2012) mantêm a estrutura fundamental desse pensamento, modificando, entretanto, a interpretação das ações e das responsabilidades que cabem a cada um dos personagens. Tem-se, então, a régua do valor criado estratificada em três partes, denominadas: a parte do fornecedor, o valor apropriado e o excedente do cliente. A parte do fornecedor diz respeito aos benefícios defasados e aos ganhos operacionais, haja vista que existem mecanismos de relacionamento com fornecedores que podem provocar melhoria no desempenho operacional (Brito \& Brito, 2012). O valor apropriado pela firma é a parte dedicada ao lucro e à rentabilidade propriamente, pois se aloca no intervalo entre preço e custo. A lucratividade deve ser ampliada, a fim de remunerar do melhor modo possível os acionistas que empregam capital na firma (Brito \& Brito, 2012), e é esta a dimensão da vantagem competitiva adotada nesta pesquisa.

Por fim, tem-se a longitudinalidade dos estudos de desempenho, que se trata não apenas de investigar os motivos que proporcionaram vantagem competitiva à firma no passado, mas também de como mantê-la com o passar dos anos. Para tanto, o ganho dessa vantagem não deve ser encarado como fruto de um fator aleatório, como o acaso ou a boa sorte, mas de estratégias pensadas especificamente com esse fim. Se um evento de boa sorte ocorrer, ele deverá ser detectado de modo que possa, na medida do possível, ser estrategicamente replicado (Brito \& Brito, 2012).

\section{CONDICIONANTES AMBIENTAIS}

A definição da indústria na análise do ambiente deve abranger com precisão quais firmas efetivamente competem entre si, pois é delas que a firma sofrerá o impacto das ações decorrentes de suas decisões estratégicas. De acordo com Murthi, Rasheed, e Goll (2013), os efeitos dos grupos estratégicos, formados com base na similaridade de efeitos das variáveis representativas das estratégias das firmas no desempenho, capturam a heterogeneidade da utilização de recursos por parte das firmas.

Para que se possa refletir sobre a composição da indústria, deve-se focar a atenção na atuação e nos resultados das firmas concorrentes (Porter, 2004), procurando antever seus próximos passos e, ainda, prever o possível impacto que elas causarão no ambiente da indústria de atuação, antecipando, dessa maneira, uma reação interna a essas demandas e a esses efeitos ambientais.

Outra justificativa para a inclusão do ambiente competitivo nos estudos do desempenho é a perspectiva de Child (1972) de que o ambiente tem sido considerado a principal fonte de restrição ao planejamento estratégico da firma. A manutenção do posicionamento competitivo da firma depende da troca que ocorre com as outras firmas, e essa dependência impõe uma restrição no direcionamento estratégico da firma, fazendo com que ela deva se 
adaptar ao contexto competitivo. As firmas devem atingir certos níveis de desempenho a fim de obterem vantagens competitivas que lhes permitam desenvolver capacidade de sobreviver. Se a firma não se adapta ao seu ambiente, as oportunidades são perdidas, os custos crescem e sua sobrevivência é ameaçada.

Conforme apontam Tarziján e Ramirez (2010), ao abordarem os efeitos da firma, da indústria e da corporação no desempenho, tanto a firma quanto a indústria têm um impacto significativo no efeito da indústria no desempenho, tendo em vista que o efeito das estratégias competitivas na lucratividade da firma é diretamente relacionado à indústria à qual a firma está vinculada.

$\mathrm{Na}$ busca por compreender o conjunto de características externas que afetam simultaneamente várias firmas concorrentes, o impacto do contexto competitivo sobre a firma deve ser considerado na análise do desempenho, afirmação que encontra justificativa na tese desenvolvida por Brito (2011), a qual pontua que, "em diferentes contextos, as empresas buscam resultados distintos [...] o desempenho organizacional é algo específico da escolha estratégica de cada empresa" (p. 56).

Quanto ao dinamismo ambiental, estreitamente relacionado à rivalidade, e sua importância sob a ótica longitudinal de análise, Simerly e Li (2000, p. 38) relatam que o aumento do dinamismo ambiental faz crescer, nos gestores e demais personagens envolvidos com os negócios da firma, a incapacidade de prever com eficiência as condições ambientais, tanto do futuro quanto do presente. Dessa maneira, como cada firma adota estratégias diferentes a fim de lidar com as mesmas exposições ambientais, infere-se que o sucesso, que pode ser parcialmente mensurado pelo desempenho, também será observado de maneira distinta, haja vista o posicionamento de cada uma diante da rivalidade ambiental (Simerly \& Li, 2000).

Segundo Simerly e Li (2000), espera-se que as características particulares do ambiente de uma indústria afetem as firmas de modo semelhante, enquanto, dentro de cada firma, o sucesso depende da adoção de mecanismos de resposta apropriados para tratar os fatores ambientais. A análise de várias pesquisas empíricas demonstra que uma maior incerteza sobre os aspectos ambientais está associada com o maior dinamismo ambiental, definido como o produto de várias forças operando em um mesmo tempo e mensurado como a taxa de instabilidade às mudanças ambientais, diretamente pelo grau de rivalidade na indústria.

\section{HIPÓTESES DE PESQUISA E MODELO DE ANÁLISE}

Em sintonia com o objetivo estabelecido para este trabalho, considerando os aspectos apontados em relação aos efeitos da indústria e da própria firma no desempenho, as hipóteses de pesquisa são:

H1: Há uma relação não linear entre o desempenho da firma e o comportamento do ambiente da indústria da qual ela faz parte.

H2: A influência do ambiente da indústria no desempenho da firma aumenta ao longo do tempo.

O modelo final de análise foi proposto tomando-se por referência as fundamentações teóricas apresentadas anteriormente, cujo cerne encontra-se descrito na abordagem teórica para vantagem competitiva desenvolvida por Brito e Brito (2012). A operacionalização desta pesquisa deu-se com os constructos validados na pesquisa desenvolvida por Dias (2004) para a lucratividade. Nesta pesquisa, as variáveis relacionadas ao ambiente da indústria foram extraídas da pesquisa de Dias (2009).

\section{METODOLOGIA}

Direcionando-se para o problema da mensuração do desempenho propriamente, os textos de Simerly e Li (2000) e de Richard et al. (2009) nos orientam para o fato que o desempenho é um constructo multidimensional e que, assim, um único índice não é suficiente para fornecer uma compreensão abrangente da relação existente entre o desempenho e os demais constructos de interesse.

Essa multidimensionalidade conceitual é apontada como um consenso por Brito e Brito (2012), na discussão sobre o desempenho da firma. A diferença dependerá, portanto, do método de análise, da fonte utilizada para obtenção dos dados e das métricas adotadas pelo pesquisador (Combs, Crook, \& Shook, 2005). Essas dimensões são úteis em posicionar pesquisas teóricas realizadas que objetivam ampliar a compreensão acerca do constructo desempenho e para definir as características desta pesquisa.

\section{Operacionalização e mensuração das variáveis}

As variáveis adotadas para a execução do modelo de pesquisa são apresentadas nesta subseção, baseadas nas pesquisas desenvolvidas por Dias $(2004,2009)$. Primeiramente, são apresentadas as variáveis representativas do ambiente da indústria para, em seguida, serem apresentadas as variáveis que integram o constructo lucratividade. 


\section{Ambiente da indústria - Variáveis preditoras}

As variáveis representativas do ambiente da indústria foram metodologicamente propostas na tese desenvolvida por Dias (2009), entretanto, para fins metodológicos, adotou-se trabalhar com as variáveis padronizadas pela distribuição normal centradas na média zero e com variância igual a um. São elas:

- RECTOT: "Variação [...] entre períodos da receita total, calculada pela soma das receitas operacionais, das receitas não operacionais e da receita líquida de vendas total” (Dias, 2009, p. 81).

- RVLIQ: “Variação [...] entre períodos das receitas oriundas das vendas líquidas, que correspondem à diferença entre a receita bruta total (proveniente da venda de produtos e serviços industriais, da revenda de mercadorias e da prestação de serviços não industriais) e o total das deduções (vendas canceladas e descontos, ICMS e outros impostos e contribuições incidentes sobre as vendas e serviços, como Cofins, Simples etc.)” (Dias, 2009, p. 81).

Tomando por base as abordagens de Porter (1979, 1981), de Tan e Litschert (1994) e de Sharp, Bergh e Li (2013) e considerando a influência da rivalidade como parte do ambiente competitivo, a rivalidade da indústria foi acessada por meio da média das variáveis RECTOT e RVLIQ das firmas concorrentes, excluindo-se a influência da própria firma no indicador, o que traz a certeza de que esse indicador calculado representará exclusivamente o impacto da estrutura da concorrência, refletindo a rivalidade do segmento da indústria em dois níveis de estratificação da North America Industrial Classification (NAICS). Tendo em vista a perspectiva de Tirole (1988) de que os preços praticados pela firma permitem uma rápida adaptação à rivalidade enfrentada no ambiente competitivo, a utilização das receitas bruta e líquida médias das firmas atuantes em uma mesma indústria como proxies da rivalidade mostra-se adequada aos objetivos desta pesquisa.

0 uso de tais variáveis como controles de quase indústria em detrimento de varáveis dummy está em alinhamento com os estudos de Sharp et al. (2013), que apontam que as variáveis dummy apresentam a limitação de influenciarem negativamente o poder estatístico do modelo de regressão, além de comprometerem a parcimônia. Além disso, ainda conforme os autores quanto ao nível de análise, é indicada a utilização de variáveis que representem a diferença entre o desempenho médio da indústria e o desempenho específico da firma.
Assim, em alinhamento com Sharp et al. (2013), tem-se as seguintes variáveis de análise, as quais permitem o controle do efeito da indústria e a mensuração de tal efeito, simultaneamente:

- RECTOT $_{\mathrm{Ni}}$ : representa o impacto da estrutura da concorrência, representada pelo RECTOT, no i-ésimo nível de segmentação da NAICS, para todo $i=01,02$.

- $R V L I Q_{N o i}:$ representa o impacto da estrutura da concorrência, representada pelo RVLIQ, no i-ésimo nível de segmentação da NAICS, para todo $i=01,02$.

O cálculo final estimado do segmento da indústria para cada firma é representado, para a variável RECTOT, genericamente pela fórmula (1).

$$
\operatorname{RECTOTN}_{l k}=\frac{\left[\sum_{l=1}^{j} \text { RECTOT }_{l}\right]-\text { RECTOT }_{k}}{j-1}
$$

E, analogamente, para a variável RVLIQ, pela fórmula (2).

$$
R \widehat{R V I Q} N_{l k}=\frac{\left[\sum_{l=1}^{j} R V L I Q_{1}\right]-R V L I Q_{k}}{j-1}
$$

Em que:

$\mathrm{k}=$ firma;

$\mathrm{j}=$ número de firmas que compõem o segmento, no i-ésimo nível da NAICS.

\section{Desempenho financeiro (lucratividade) - Constructo resposta}

As variáveis constituintes do constructo desempenho, associado à lucratividade da firma, foram metodologicamente propostas na dissertação desenvolvida por Santos (2008). São elas:

- $\quad$ ROA - retorno sobre o ativo.

- $\quad$ EBITDA - margem de lucro antes dos impostos, da depreciação e da amortização.

- $\quad \mathrm{ROI}$ - retorno sobre o investimento.

- ROS - retorno sobre as vendas.

- ROE - retorno sobre o patrimônio líquido.

\section{Coleta dos dados}

Foram consideradas unidades de observação firmas brasileiras de capital aberto, ativas no período de 2005 a 2012, que apresentaram informações contábeis na base Economática ${ }^{\circ}$, publicaram informações trimestrais na base da Comissão de Valores 
Mobiliários e tiveram ações negociadas em bolsa nesses mesmos anos. A adoção desse intervalo para a pesquisa deu-se por motivos técnicos, devido à disponibilidade das variáveis consideradas nesta pesquisa, de modo que o tamanho da amostra não ficasse comprometido, e, principalmente, em função da crise econômica ocorrida em 2008, nos Estados Unidos, englobando tanto períodos que a precederam (de 2005 a 2007) quanto que a sucederam (de 2009 a 2012), assim como o próprio ano de 2008, cujos reflexos, com diferentes intensidades, alcançaram âmbito mundial.

Foram excluídas da amostra inicial as observações que se encaixaram em uma das seguintes características: (1) foram ponto de influência no cálculo da distância de Mahalanobis para amostras multivariadas (Barnett \& Lewis, 1978); (2) surgiram como ponto de influência (outlier) ao longo do processo de modelagem, via análise de regressão múltipla (Neter, Kutner, Nachtsheim, \& Wasserman, 1996); e (3) foram detectados visualmente na análise de resíduos (Barnett \& Lewis, 1978).

A exclusão de tais observações também levou em conta a proposta de Hawawini, Subramanian, e Verdin (2005), que sugerem que firmas as quais tenham obtido patamares de desempenho destacados somente devem ser mantidas na amostra caso, em conjunto e de maneira significativa, isso ocorra de modo persistente durante o período sob análise, fato que representa a manutenção de vantagens competitivas sustentadas.

Constituíram a amostra final de 178 (2005) a 252 (2012) firmas, conforme Tabela 1, e a identificação e o agrupamento das firmas por indústria foram efetivados com base nos níveis um e dois da NAICS.

Tabela 1. Composição das amostras por ano

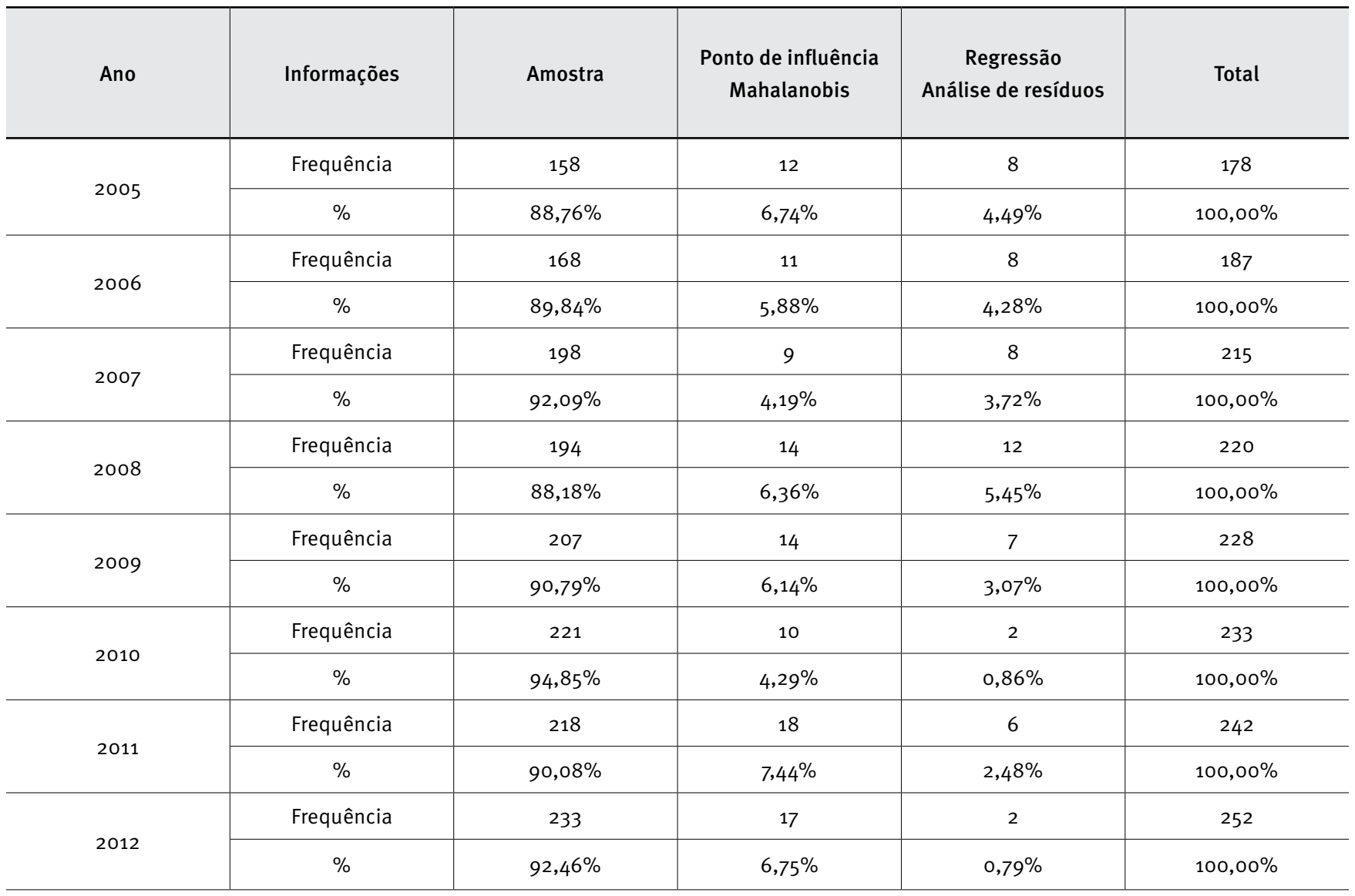

\section{APRESENTAÇÃO, TRATAMENTO DOS DADOS E ANÁLISE DOS RESULTADOS}

Considerando o enfoque da pesquisa que avalia o impacto dos diferentes níveis de estratificação do ambiente da indústria no desempenho da firma, os resultados da análise descritiva consideram essa estratificação por níveis da indústria. As principais etapas metodológicas seguidas com o objetivo de verificar as hipóteses de pesquisa foram: 
a) estimar, para o período mais recente, a composição dos constructos constituintes do modelo, em termos do peso atribuído a cada uma das variáveis, via análise de componentes principais;

b) mensurar o percentual da variância total do construto resposta lucratividade em função das variáveis representativas do ambiente da indústria, por meio do cálculo da decomposição da variância, oriundo da análise de variância obtida a partir do modelo de regressão completo. Essa análise foi efetuada para todos os períodos considerados na pesquisa, e os resultados obtidos foram comparados longitudinalmente, a fim de se identificar uma possível persistência da explicação da variância. O tamanho médio dos concorrentes foi incluído no modelo como variável de controle;

c) verificar, para o período mais recente considerado na análise, a significância das relações entre as variáveis representativas da rivalidade na indústria e o constructo lucratividade, adotado como indicador do desempenho da firma, delineando o comportamento dessas relações via análise de regressão. Depois de construído o modelo para o período mais recente, ele foi replicado para os períodos anteriores. Os resultados obtidos foram comparados longitudinalmente, a fim de se verificar a persistência da capacidade explicativa do modelo, ao longo do tempo.

Posteriormente à apresentação dos resultados da estatística descritiva estratificados por níveis da indústria, o constructo lucratividade teve sua composição definida via análise de componentes principais, por meio da adoção da primeira componente construída, e, em seguida, o impacto de cada uma das variáveis do ambiente da indústria sobre esse constructo foi testado.

Haja vista o caráter longitudinal comparativo desta pesquisa, o modelo que associou o constructo representativo do desempenho às variáveis do ambiente da indústria foi obtido via análise de regressão múltipla para o período mais recente, ou seja, para o ano de 2012. A adequação da utilização de tal método está alinhada com a pesquisa de Bamiatzi, Bozos, e Nikolopoulos (2010).

Em seguida, esse mesmo modelo foi aplicado aos períodos anteriores, e os resultados foram comparados ano a ano, por meio da evolução da proporção da variância explicada, por variável. Os modelos de regressão pesquisados testaram, além da linearidade, a possível existência da não linearidade, por meio da inclusão do termo quadrático no processo de modelagem.

Para todos os modelos obtidos por meio de equações de regressão, foram confirmadas as suposições de normalidade e homocedasticidade, via análise de resíduos (Neter et al., 1996), validando os modelos construídos. Já a suposição de autocorrelação dos resíduos não foi considerada nesses modelos, uma vez que os dados não foram obtidos de modo sequencial em um mesmo processo, mas, sim, como resultados oriundos de diferentes firmas, tornando a ordem dos dados e, consequentemente, esse passo da análise dispensáveis. Para todos os casos, considerou-se um nível de significância de $5 \%$ $(\alpha=0,05)$.

\section{Ambiente da indústria}

Esta subseção detém-se em descrever o comportamento do ambiente da indústria por meio das variáveis RECTOT e RVLIQ ao longo dos oito anos considerados na análise. Todas as variáveis foram padronizadas, e, na Tabela 2, encontram-se as estatísticas descritivas que sumarizam o comportamento da amostra para a variável RECTOT constituídas por média, desvio padrão, mínimo, primeiro quartil (Q1), segundo quartil ou mediana, terceiro quartil (Q3) e máximo.

Tabela 2. Estatísticas descritivas - RECTOT (padronizado) de 2005 a 2012

\begin{tabular}{|c|c|c|c|c|c|c|c|}
\hline Ano & Média & Desvio padrão & Mínimo & $Q_{1}$ & Mediana & Q3 & Máximo \\
\hline 2007 & $-0,081$ & 0,336 & $-0,239$ & $-0,230$ & $-0,198$ & $-0,088$ & 2,472 \\
\hline 2008 & $-0,027$ & 0,440 & $-0,234$ & $-0,212$ & $-0,178$ & $-0,043$ & 2,753 \\
\hline 2010 & 0,005 & 0,509 & $-0,265$ & $-0,237$ & $-0,180$ & $-0,003$ & 3,637 \\
\hline 2011 & $-0,045$ & 0,397 & $-0,256$ & $-0,230$ & $-0,186$ & $-0,053$ & 2,603 \\
\hline 2012 & $-0,019$ & 0,498 & $-0,258$ & $-0,234$ & $-0,184$ & $-0,049$ & 3,846 \\
\hline
\end{tabular}


A análise de variância aponta para uma constância no efeito do ambiente mensurado pela variável RECTOT, cujo resultado apontou para a não significância da diferença entre os anos (P-Valor = 0,678). 0 ano de 2007 apresentou um resultado diferente em relação aos demais, que reflete apenas o comportamento da amostra e não representa significância estatística. Na Tabela 3, encontram-se as estatísticas descritivas que sumarizam o comportamento da amostra para a variável RVLIQ.

Tabela 3. Estatísticas descritivas - RVLIQ (padronizado) de 2005 a 2012

\begin{tabular}{c|c|c|c|c|c|c|c}
\hline Ano & Média & Desvio padrão & Mínimo & Q1 & Mediana & Q3 & Máximo \\
\hline 2005 & $-0,034$ & 0,473 & $-0,231$ & $-0,213$ & $-0,180$ & $-0,037$ & 4,506 \\
\hline 2006 & $-0,045$ & 0,409 & $-0,231$ & $-0,208$ & $-0,172$ & $-0,017$ & 4,194 \\
\hline 2007 & $-0,091$ & 0,304 & $-0,229$ & $-0,219$ & $-0,194$ & $-0,090$ & 2,531 \\
\hline 2008 & $-0,040$ & 0,512 & $-0,231$ & $-0,212$ & $-0,177$ & $-0,075$ & 5,377 \\
\hline 2009 & $-0,031$ & 0,480 & $-0,238$ & $-0,206$ & $-0,161$ & $-0,035$ & 5,120 \\
\hline 2010 & $-0,020$ & 0,455 & $-0,244$ & $-0,210$ & $-0,167$ & 0,006 & 4,365 \\
\hline 2011 & $-0,059$ & 0,390 & $-0,239$ & $-0,201$ & $-0,165$ & $-0,053$ & 4,485 \\
\hline 2012 & $-0,055$ & 0,394 & $-0,407$ & $-0,235$ & $-0,183$ & $-0,056$ & 2,955 \\
\hline
\end{tabular}

A exemplo dos resultados apresentados pela variável RECTOT, a análise de variância também aponta para uma constância no efeito do ambiente mensurado pela variável RVLIQ, com resultado não significativo para a diferença entre os anos (P-Valor $=0,845)$. Novamente, o ano de 2007 apresentou um resultado diferente em relação aos demais, sendo este apenas reflexo do comportamento da amostra.

\section{Lucratividade}

O constructo lucratividade, como parte representativa do desempenho da firma é, nesta pesquisa, composto pelas variáveis: retorno sobre o ativo (ROA), margem de lucro líquido antes do imposto, depreciação e amortização (EBITDA), retorno sobre o investimento (ROI), retorno sobre as vendas (ROS) e retorno sobre o patrimônio líquido (ROE).

Para que se possa identificar a composição mais adequada do constructo lucratividade, considerando a variância do vetor composto pelas cinco variáveis mencionadas, efetuou-se uma análise de componentes principais. A função definida pela primeira componente principal ( $\left.\mathrm{CP}_{1}\right)$ obtida, que apresenta a maior proporção de variância explicada, foi escolhida como representativa do constructo lucratividade.

Essa análise foi replicada para todos os anos da pesquisa, e a estabilidade longitudinal da composição do constructo, bem como a importância que cada variável possui na composição da lucratividade associada ao seu peso de inclusão na função obtida por meio da primeira componente principal construída, é apresentada na Tabela 4.

Tabela 4. Evolução longitudinal da composição do constructo lucratividade - 1a componente principal de cada ano

\begin{tabular}{c|c|c|c|c|c|c|c|c}
\hline Variável & $\mathbf{2 0 0 5}$ & $\mathbf{2 0 0 6}$ & $\mathbf{2 0 0 7}$ & $\mathbf{2 0 0 8}$ & $\mathbf{2 0 0 9}$ & $\mathbf{2 0 1 0}$ & $\mathbf{2 0 1 1}$ & $\mathbf{2 0 1 2}$ \\
\hline ROA & 0,602 & 0,616 & 0,522 & 0,619 & 0,573 & 0,629 & 0,594 & 0,601 \\
\hline EBITDA & 0,129 & 0,161 & 0,077 & 0,159 & 0,092 & 0,105 & 0,118 & 0,109 \\
\hline ROI & 0,553 & 0,519 & 0,519 & 0,547 & 0,561 & 0,483 & 0,567 & 0,566 \\
\hline ROS & 0,109 & 0,002 & 0,056 & 0,057 & 0,008 & 0,034 & 0,013 & 0,105 \\
\hline ROE & 0,551 & 0,570 & 0,670 & 0,538 & 0,590 & 0,599 & 0,559 & 0,543 \\
\hline Autovalor & 2,544 & 2,322 & 1,796 & 2,272 & 2,248 & 2,282 & 2,575 & 2,524 \\
\hline $\begin{array}{c}\text { Proporção de } \\
\text { explicabilidade }\end{array}$ & 0,509 & 0,464 & 0,359 & 0,454 & 0,450 & 0,456 & 0,515 & 0,505 \\
\hline $\begin{array}{c}\text { Proporção } \\
\text { acumulada }\end{array}$ & 0,509 & 0,464 & 0,359 & 0,454 & 0,450 & 0,456 & 0,515 & 0,505 \\
\hline
\end{tabular}


As variáveis de maior peso na composição do constructo lucratividade foram o ROA, o ROI e o ROE, que apresentaram flutuações de peso entre 0,483 (variável ROI, 2010) e 0,629 (variável ROA, 2010).

No ano de 2005 , as firmas encontravam-se em um nível elevado de lucratividade, se comparado aos demais anos da pesquisa, porém com alta variabilidade. No ano seguinte, esse patamar médio manteve-se, e, além disso, houve uma redução da variabilidade. Em 2007, houve uma forte queda, recuperada em 2008, mas sentida novamente em 2009. Essa flutuação coletiva pode ter apontado o início da crise e a alta volatilidade do mercado no período. A partir de 2008 , houve uma retomada gradual da lucratividade que ocorreu inicialmente com alta variabilidade, que se reduziu com o passar dos anos até 2012. Após o impacto da crise financeira, em 2009, as firmas, em média, recuperaram-se já no ano seguinte (2010), porém a recuperação não ocorreu no mesmo ritmo para todas. Nos anos seguintes, em 2011 e 2012, o comportamento de retomada manteve-se, e, finalmente, em 2012, as firmas voltaram ao patamar de lucratividade de 2005, mas com menor flutuação nos resultados. A análise de variância (ANOVA) confirma que, ao longo dos oito anos da pesquisa, a diferença nos resultados da lucratividade apresentou significância estatística (P-Valor = 0,000).

A comparação múltipla de Tukey, apresentada na Tabela 5, permite detectar exatamente quais são os pares de anos cuja lucratividade média foi significativamente diferente e respalda as conclusões anteriores.

\section{Tabela 5. Comparação múltipla de Tukey - lucratividade de 2005 a 2012}

\begin{tabular}{|c|c|c|c|c|c|}
\hline \multirow{2}{*}{\multicolumn{2}{|c|}{ Anos comparados }} & \multicolumn{3}{|c|}{ Intervalo e confiança (95\%) para a diferença entre os anos } & \multirow{3}{*}{$\frac{\text { Conclusão }}{\text { Igual }}$} \\
\hline & & \multirow{2}{*}{$\begin{array}{c}\text { Limite inferior } \\
-0,0738\end{array}$} & \multirow{2}{*}{ Média } & \multirow{2}{*}{$\begin{array}{c}\text { Limite superior } \\
0,1495\end{array}$} & \\
\hline \multirow{7}{*}{2005} & 2006 & & & & \\
\hline & 2007 & $-0,2337$ & $-0,1263$ & $-0,0190$ & Diferente \\
\hline & 2008 & $-0,0800$ & 0,0274 & 0,1349 & Igual \\
\hline & 2009 & $-0,2294$ & $-0,1232$ & $-0,0169$ & Diferente \\
\hline & 2010 & $-0,1720$ & $-0,0670$ & 0,0381 & Igual \\
\hline & 2011 & $-0,1040$ & 0,0013 & 0,1065 & Igual \\
\hline & 2012 & $-0,0586$ & 0,0454 & 0,1493 & Igual \\
\hline \multirow{6}{*}{2006} & 2007 & $-0,2713$ & $-0,1641$ & $-0,0570$ & Diferente \\
\hline & 2008 & $-0,1177$ & $-0,0104$ & 0,0969 & Igual \\
\hline & 2009 & $-0,2671$ & $-0,1610$ & $-0,0549$ & Diferente \\
\hline & 2010 & $-0,2097$ & $-0,1048$ & 0,0001 & Igual \\
\hline & 2011 & $-0,1416$ & $-0,0365$ & 0,0685 & Igual \\
\hline & 2012 & $-0,0962$ & 0,0076 & 0,1113 & Igual \\
\hline \multirow{5}{*}{2007} & 2008 & 0,0510 & 0,1537 & 0,2565 & Diferente \\
\hline & 2009 & $-0,0984$ & 0,0031 & 0,1047 & Igual \\
\hline & 2010 & $-0,0409$ & 0,0593 & 0,1596 & Igual \\
\hline & 2011 & 0,0271 & 0,1276 & 0,2281 & Diferente \\
\hline & 2012 & 0,0726 & 0,1717 & 0,2708 & Diferente \\
\hline \multirow{4}{*}{2008} & 2009 & $-0,2523$ & $-0,1506$ & $-0,0490$ & Diferente \\
\hline & 2010 & $-0,1948$ & $-0,0944$ & 0,0060 & Igual \\
\hline & 2011 & $-0,1268$ & $-0,0261$ & 0,0745 & Igual \\
\hline & 2012 & $-0,0813$ & 0,0179 & 0,1172 & Igual \\
\hline \multirow{3}{*}{2009} & 2010 & $-0,0429$ & 0,0562 & 0,1553 & Igual \\
\hline & 2011 & 0,0251 & 0,1245 & 0,2238 & Diferente \\
\hline & 2012 & 0,0706 & 0,1686 & 0,2665 & Diferente \\
\hline \multirow{2}{*}{2010} & 2011 & $-0,0298$ & 0,0683 & 0,1663 & Igual \\
\hline & 2012 & 0,0157 & 0,1123 & 0,2090 & Diferente \\
\hline 2011 & 2012 & $-0,0528$ & 0,0441 & 0,1409 & Igual \\
\hline
\end{tabular}


Modelo empírico lucratividade vs. ambiente da indústria

O impacto que o ambiente da indústria exerce sobre a lucratividade da firma é mensurado em função das variáveis RECTOT e RVLIQ, além da média dos concorrentes de cada firma que atuem no mesmo nível 1 da indústria (RECTOT ${ }_{\text {No1 }}$ e RVLIQ ${ }_{\mathrm{No} 1}$ ) e nível 2 da indústria $\left(\mathrm{RECTOT}_{\mathrm{No2}}\right.$ e $\mathrm{RVLI}_{\mathrm{NO} 2}$ ). Todas as variáveis relacionadas ao ambiente da indústria foram padronizadas. 0 modelo de regressão principal construído foi delineado para o período mais recente, pelo fato de a dinâmica do ambiente desse ano ser a mais atual possível. Depois de obtido o modelo ótimo para esse ano, ele foi replicado para os sete anos anteriores, e a persistência da capacidade em explicar a variabilidade do constructo lucratividade, por meio das variáveis do ambiente da indústria, foi avaliada comparando-se os resultados obtidos ano a ano.

Ao longo do processo de modelagem, construíram-se os modelos de regressão apresentados na Tabela 6 .

Tabela 6. Modelos de regressão construídos ao longo da modelagem

\begin{tabular}{c|c|c|c|c|c}
\hline \multirow{2}{*}{ Fonte de variação } & \multicolumn{4}{|c}{ Modelo de regressão } \\
\cline { 2 - 6 } & Linear & Quadrático & Cúbico & Exponencial & Logarítmico \\
\hline F & 5,810 & 11,630 & 13,430 & 6,460 & 0,000 \\
\hline P-Valor & 0,000 & 0,000 & 0,000 & 1,390 & 0,245 \\
\hline$R^{2}$ (ajustado) & $13,28 \%$ & $21,93 \%$ & $27,71 \%$ & $9,33 \%$ \\
\hline
\end{tabular}

A análise exclusiva desses parâmetros induziria a escolha do modelo cúbico como o de melhor ajuste, entretanto, comparado ao modelo quadrático, o modelo cúbico apresentou forte multicolinearidade (modelo quadrático: $\mathrm{VIF}\left(\mathrm{RVLIQ}^{2}\right)=5,09$; modelo cúbico: VIF $\left.\left(\mathrm{RVLIQ}^{2}\right)=69,61 ; \mathrm{VIF}\left(\mathrm{RVLIQ}^{3}\right)=44,72\right)$, o que compromete a sua robustez, bem como sua adoção. Dessa maneira, optou-se pela adoção do modelo quadrático como o de melhor ajuste, em alinhamento com o proposto por Neter et al.(1996).

Após as simulações computacionais terem sido efetuadas, o modelo no qual estão incluídas as variáveis RVLIQ e RVLIQ2 foi identificado como o mais bem ajustado, apontando para o efeito quadrático dessa variável quando associada à lucratividade; para o efeito linear da variável de controle tamanho; e, por fim, houve, ainda, a inclusão da variável RECTOT numa relação linear, em procedimento similar ao adotado por Schilke (2014). Esse modelo apresentou capacidade explicativa acima de $20 \%$ para os dados do ano de 2012. A Tabela 7 apresenta o modelo em que se excluem as variáveis não significativas, também representado na Equação 1.

$$
\begin{aligned}
& \text { LUCRATIVIDADE } \\
& \text { 1, 0684- 0, 0602 } \\
& 0,1946 \text { RECTOT }_{N_{01}}+0,1697 \text { RVLIQ }_{N 01_{i}}-0,2454 \mathrm{RVLIQ}_{i}^{2}+\varepsilon
\end{aligned}
$$

Tabela 7. Análise de regressão lucratividade vs. ambiente da indústria - modelo final - 2012

\begin{tabular}{c|c|c|c|c}
\hline Variável preditora & Coeficiente & Erro-padrão & Estatística T & P-valor \\
\hline Constante & 1,0684 & 0,2042 & 5,23 & 0,000 \\
\hline TAMANHO & $-0,0602$ & 0,0120 & $-4,31$ & 0,000 \\
\hline RECTOT & $-0,1722$ & 0,0574 & $-3,00$ & 0,003 \\
\hline RVLIQ & 0,8587 & 0,1298 & $-0,61$ & 0,000 \\
\hline RECTOTNO1 & $-0,1946$ & 0,0714 & $-2,73$ & 0,018 \\
\hline RVLIQNO1 & 0,1697 & 0,0714 & 2,38 & $-5,18$ \\
\hline RVLIQ2 & $-0,2454$ & 0,0474 & \\
\hline
\end{tabular}

$S=0,237092 R_{2}=24,0 \% R_{2}(\operatorname{adj})=21,9 \%$ 
No modelo construído, observamos, além da contribuição das variáveis RECTOT e RVLIQ, que a variável RVLIQ foi incluída em sua composição quadrática, apontando para a existência de uma relação não linear entre a lucratividade e a receita de vendas líquida, em forma de $U$ invertido, tendo em vista o sinal negativo do parâmetro estimado, resultado similar ao apurado por Schilke (2014), fornecendo embasamento empírico para a não rejeição da hipótese de pesquisa 1 . Além disso, as duas variáveis representativas dos resultados médios do nível 1 da indústria, RECTOT $_{\text {No1 }}$ e RVLIQ ${ }_{\text {No1, }}$ também foram mantidas no modelo, indicando a influência do desempenho dos concorrentes que atuam no mesmo setor, no desempenho da firma. Considerando que o período de 2012 representa com maior fidedignidade as condições atuais do ambiente da indústria, o modelo apresentado na Equação 1 foi adotado como o modelo final para representar a lucratividade, em função das características do ambiente da indústria. Ele foi replicado para todos os sete anos anteriores que compõem a amostra da pesquisa, a fim de se comparar a sua eficácia, também nos anos anteriores.

\section{Análise longitudinal do modelo empírico}

Nesta subseção, são apresentados os resultados da estimação do modelo de referência em relação aos demais períodos que compuseram o estudo. As mesmas variáveis significativas que compuseram o modelo de regressão estimador da lucratividade em função das variáveis do ambiente da indústria foram incluídas em outras regressões, a fim de simular a flutuação no comportamento destas, porém para os sete anos anteriores da pesquisa.

A longitudinalidade dos estudos de desempenho trata não apenas de investigar os motivos que trouxeram vantagem competitiva à firma no passado, mas, também, de como mantê-la com o passar dos anos. Para tanto, o ganho dessa vantagem não deve ser encarado como fruto de um fator aleatório, como o acaso ou a boa sorte, mas de estratégias pensadas especificamente com esse fim.

A persistência do desempenho foi definida por Waring (1996) em um modelo de série temporal autorregressivo de ordem $1(A R(1))$. Esse tipo de análise acompanha os resultados de cada item da amostra, ou seja, o desempenho de cada firma linearmente, com o passar do tempo. Entretanto, os modelos de série-temporal apresentam algumas suposições, sendo a sazonalidade a principal delas, pois é por meio do estudo probabilístico da repetição do comportamento que a construção do modelo empírico torna-se viável, o que não foi possível neste caso.

Haja vista a ausência de sazonalidade, construir um modelo para cada espaço de tempo, considerando o comportamento da amostra como um todo e comparando-os em seguida, parece ser mais razoável do que trabalhar com a média, por firma, dos vários momentos presentes na amostra, pois a média dilui o efeito do tempo e, logo, faz supor que eles são oriundos de uma mesma realidade histórica, o que não faz sentido, em se tratando de estratégia organizacional.

Para tanto, optou-se por replicar o modelo construído para o período mais recente aos sete anos anteriores. A principal vantagem dessa abordagem sobre a inclusão dos anos no modelo de regressão como variáveis dummy vem do fato de que assim é possível efetuar a decomposição da variância anualmente, o que não seria possível caso todos os anos fossem incluídos em uma única equação de regressão, possibilitando uma comparação anual da influência proporcional do ambiente da indústria. Além disso, um modelo com oito variáveis dummy para cada período seria pouco parcimonioso e suporia que o comportamento não linear seria o mesmo para todos os anos, o que de fato não ocorre.

O modelo construído para o ano de 2005 manteve as variáveis RECTOT e RVLIQ, incluindo o termo quadrático, como significativas. 0 total da variância explicada foi de apenas 8,30\%, porém o modelo apresentou significância estatística ( $\mathrm{P}$-Valor = 0,000) e pode ser considerado válido. 0 modelo construído para o ano de 2006 também manteve as variáveis RECTOT e RVLIQ, incluindo o termo quadrático, como significativas, apontando para a estabilidade no comportamento do ambiente da indústria representado pela rivalidade nesses dois anos. 0 total da variância explicada foi de $13,10 \%$, superior ao ano anterior, e o modelo novamente apresentou significância estatística ( $P$-Valor =,000), podendo ser considerado válido.

Já o modelo construído para o ano de 2007 manteve apenas a variável $\mathrm{RVLIQ}_{\mathrm{No1}}$ como significativa. O total da variância explicada foi de $7,70 \%$, apontando para uma queda na capacidade explicativa do modelo. Entretanto, o modelo é válido em estimar parte da variabilidade da lucratividade (P-Valor $=0,003)$. Considerando a natureza polinomial da equação de regressão, mesmo não havendo significância do termo RVLIQ, este deve ser mantido no modelo a fim de manter o polinômio completo, haja vista a significância da variável RVLIQ².

Para o ano de 2008, o modelo de regressão manteve as seguintes variáveis: RVLIQ, incluindo seu efeito quadrático RVLIQ², e as duas variáveis representativas do comportamento médio do setor (RECTOT No1 $_{\text {e RVLIQ }}$ ) ). 0 total da variância explicada foi de $19,20 \%$, apontando para uma capacidade explicativa muito próxima àquela observada no modelo original de 2012. No ano de 2009, 0 modelo de regressão apresentou significância das variáveis RVLIQ, incluindo seu efeito quadrático RVLIQ², e RECTOT. O total da variância explicada foi de $12,20 \%$. No ano de 2010 , o modelo de regressão apresentou significância para a constante, a variável de controle 
tamanho, as variáveis RVLIQ, incluindo seu efeito quadrático RVLIQ², e RECTOT. 0 total da variância explicada foi de $16,90 \%$.

Para o ano de 2011, o modelo de regressão apresentou significância para os seguintes termos: a constante, a variável de controle tamanho, as variáveis RVLIQ, incluindo seu efeito quadrático RVLIQ 2 , e RECTOT. O total da variância explicada foi de $10,00 \%$.

A seguir, na Tabela 8, tem-se a composição das equações de regressão construídas para os anos da pesquisa, incluídos os termos do modelo original construído para o ano de 2012.

Tabela 8. Coeficientes dos modelos - regressão lucratividade - de 2005 a 2012

\begin{tabular}{|c|c|c|c|c|c|c|c|c|}
\hline Variável preditora & 2005 & 2006 & 2007 & 2008 & 2009 & 2010 & 2011 & 2012 \\
\hline Constante & 0,7139 & $-0,0896$ & $-0,0161$ & 0,3308 & $-0,2317$ & 0,9201 & 1,1278 & 1,0684 \\
\hline TAMANHO & $-0,0364$ & 0,0215 & 0,0037 & $-0,0128$ & 0,0175 & $-0,0574$ & $-0,0643$ & $-0,0602$ \\
\hline RECTOT & $-0,6710$ & $-0,4644$ & 0,0895 & $-0,0683$ & $-0,1833$ & $-0,3316$ & $-0,6247$ & $-0,1722$ \\
\hline RVLIQ & 1,7390 & 1,0498 & $-0,0996$ & 0,4418 & 0,5058 & 1,2441 & 1,7337 & 0,8587 \\
\hline RECTOT $_{\mathrm{No1}}$ & $-0,0266$ & 0,5015 & $-0,1262$ & $-0,2442$ & 0,0233 & 0,0953 & $-0,1000$ & $-0,1946$ \\
\hline RVLIQ $_{\mathrm{No1}}$ & 0,0172 & $-0,5380$ & 0,5275 & 0,2432 & $-0,0380$ & $-0,1980$ & 0,0913 & 0,1697 \\
\hline $\mathrm{RVLIQ}^{2}$ & $-0,3350$ & $-0,2113$ & 0,0500 & $-0,0694$ & $-0,0923$ & $-0,2614$ & $-0,3338$ & $-0,2454$ \\
\hline $\mathrm{R}_{\text {adj }}^{2}$ & $8,30 \%$ & $13,10 \%$ & $7,70 \%$ & $19,20 \%$ & $12,20 \%$ & $16,90 \%$ & $10,00 \%$ & $21,90 \%$ \\
\hline
\end{tabular}

A Tabela 9 representa a decomposição da variância, por ano, em termos percentuais, extraída a partir da soma de quadrados da ANOVA, associada ao modelo de regressão completo construído para todos os períodos. Esse resultado demonstra exatamente em quanto cada variável estudada contribui para a explicação da variância total do constructo lucratividade, e o resultado sumarizado é apresentado no Gráfico 1, fornecendo embasamento empírico para a não rejeição da hipótese de pesquisa 2.

Tabela 9. Decomposição da variância (\%) - regressão lucratividade - de 2005 a 2012

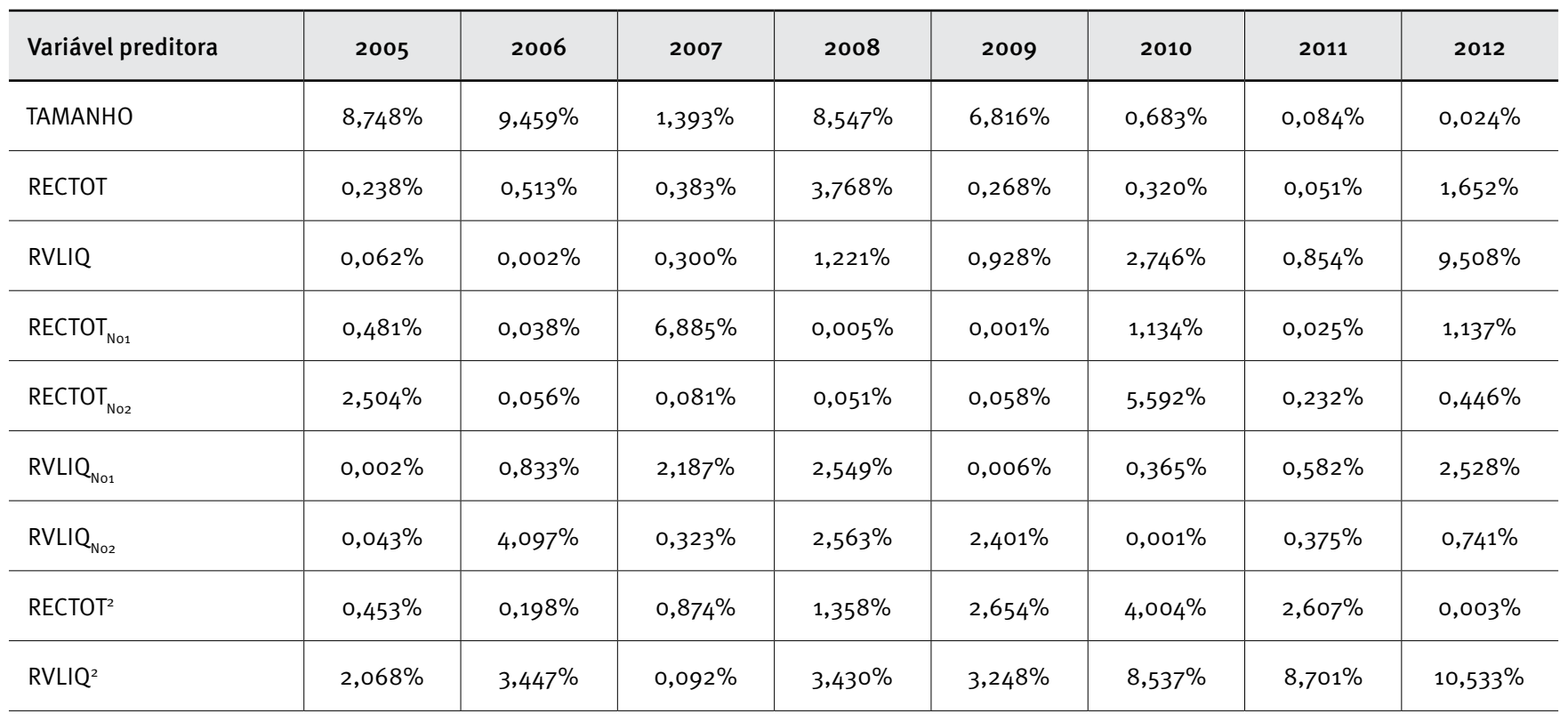


Gráfico 1. Decomposição da variância (em \%) - regressão lucratividade - de 2005 a 2012

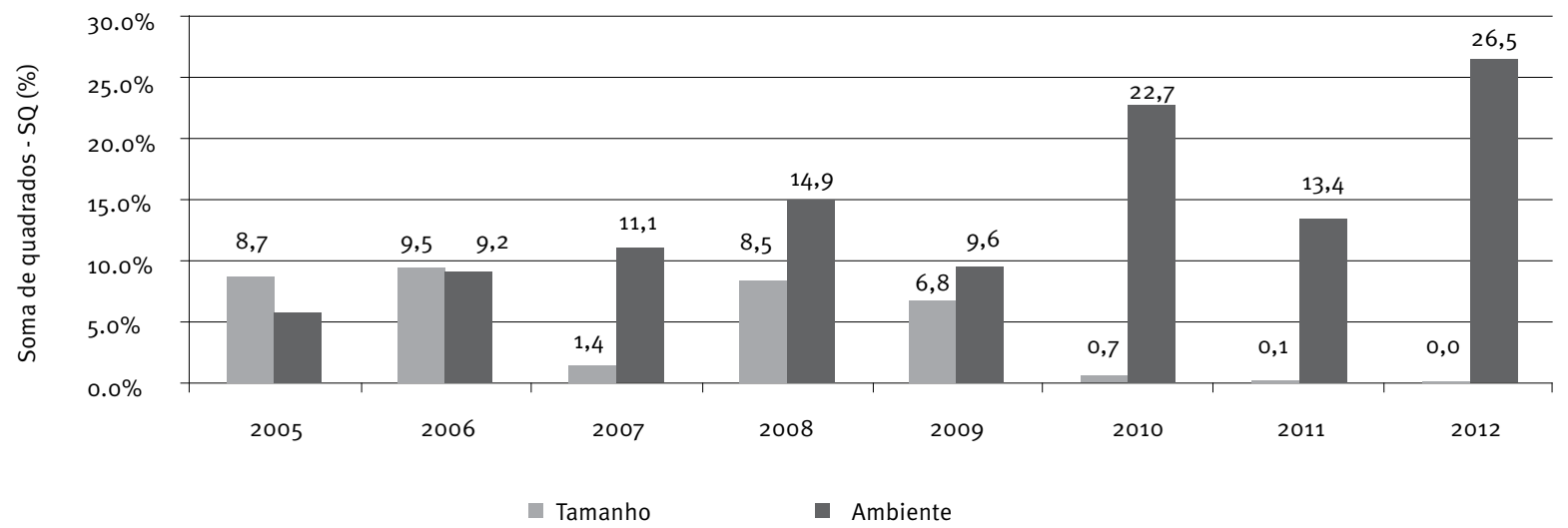

Houve uma grande flutuação na capacidade explicativa do modelo construído para estimar a lucratividade em função das variáveis do ambiente da indústria, tendo em vista o modelo inicial construído para o ano de 2012.

Em relação aos modelos matemáticos representados pelas equações de regressão construídas, propriamente, não há a indicação de ascensão do $\mathrm{R}^{2}$ com o passar dos anos, o que aponta para a impossibilidade de construir um modelo matemático único ou, ainda, que inclua um possível efeito do tempo, já que esse efeito varia aleatoriamente em termos de capacidade de explicação da variância da variável resposta, para a equação avaliada neste estudo.

Entretanto, a análise longitudinal comparativa das oito equações de regressão obtidas aponta para uma semelhança fundamental entre elas: a quase constância da inclusão da variável RECTOT em sua representação linear e da RVLIQ em sua representação quadrática, o que sugere uma tendência clara de relação entre a lucratividade e essas variáveis representativas do ambiente da indústria.

\section{CONSIDERAÇÕES E CONCLUSÕES}

Como resultado do esforço por contribuir para a compreensão das relações entre contexto competitivo e desempenho da firma, este trabalho apresenta contribuições de naturezas metodológica e acadêmica. A contribuição metodológica reside na proposição de um modelo de regressão múltipla não linear quadrático que aborda a influência que o ambiente da indústria exerce sobre o desempenho representado em termos da lucratividade da firma.
Além de se mensurarem múltiplos itens ao compor o constructo lucratividade por meio da análise de componentes principais, pôde-se adotá-lo como variável resposta em um modelo de regressão. Essa escolha possibilitou, além de identificar a significância dos efeitos das variáveis representativas da indústria no desempenho, quantificar o efeito de cada uma delas sobre o constructo resposta, bem como investigar o comportamento dessas variáveis mais a fundo, com a inclusão do termo quadrático nas variáveis representativas do ambiente da indústria, constituindo a contribuição acadêmica da pesquisa.

A relação quadrática identificada fornece embasamento empírico que sustenta a hipótese de pesquisa 1, pois aponta para a existência de um ponto de equilíbrio da variável RVLIQ que, caso ultrapassado, provoca efeito inverso na lucratividade, podendo, até mesmo, culminar em prejuízo para a firma e redução ou eliminação de vantagens competitivas. Dessa forma, além de identificar o efeito crescente da rivalidade na indústria sobre a lucratividade, o estudo focou a importância da definição qualificada das ações estratégicas, pois o efeito sobre a lucratividade será nulo, e, portanto, a firma não conseguirá criar vantagem competitiva, caso se mantenha atuando na média das demais, constatação que constitui a contribuição gerencial desta pesquisa. Esses resultados estão em alinhamento com a perspectiva de que, quanto maior a rivalidade, mensurada por meio da receita dos concorrentes, maior a possibilidade de obtenção de receitas originadas da venda de produtos, até o ponto em que a intensidade da rivalidade passa a dificultar 0 acesso a recursos oriundos das vendas, em função do incremento da capacidade competitiva dos concorrentes, quando comparada à capacidade competitiva da firma em foco. 
Sob a ótica longitudinal, conforme proposto na hipótese 2, fazendo-se uso da decomposição da variância, foi identificada tendência de incremento quanto ao efeito do ambiente da indústria sobre o desempenho da firma, desde os menores 5,90\% de variabilidade da lucratividade explicada pelo ambiente da indústria em 2005, até 26,50\% para o período mais recente (2012).

Como principal limitação deste estudo, tem-se a não inclusão de firmas brasileiras de capital fechado na composição da amostra, devido à não disponibilidade de informações necessárias para a operacionalização do constructo lucratividade, o que dificulta a generalização dos resultados e aponta para o desenvolvimento de pesquisas que incluam firmas brasileiras de capital fechado. Outra sugestão de pesquisa é a inclusão de variáveis que representem outras características do ambiente da indústria, além da rivalidade.

\section{REFERÊNCIAS}

Bamiatzi, V., Bozos, K., \& Nikolopoulos, K. (2010). On the predictability of firm performance via simple time-series and econometric models: Evidence from UK SMEs. Applied Economics Letters, 17(3), 279-282. doi:10.1080/13504850701720163

Bamiatzi, V., \& Hall, G. (2009). Firm versus sector effects on profitability and growth: The importance of size and interaction. International Journal of the Economics of Business, 16(2), 205-220. doi:10.1080/13571510902917517

Barnett, V., \& Lewis, T. (1978). Outliers in statistical data. Bath, EUA: John Wiley \& Sons.

Brito, R. P. (2011). Criação de valor, vantagem competitiva e seu efeito no desempenho financeiro das empresas (Tese de doutorado, Escola de Administração de Empresas de São Paulo da Fundação Getulio Vargas).

Brito, R. P., \& Brito, L. A. L. (2012). Vantagem competitiva, criação e valor e seus efeitos sobre o desempenho. RAE-Revista de Administração de Empresas, 52(1), 70-84. doi:10.1590/So034-75902012000100006

Cameron, K. S., \& Whetten, D. A. (1983). Organizational effectiveness: A comparison of multiples models. San Diego, EUA: Academic Press.

Child, J. (1972). Organizational structure, environment and performance: The role of strategic choice. Sociology, 6(1), 1-22. doi:10.1177/003803857200600101

Child, J. (1974). What determines organization performance? The universals vs. the it-all-depends. Organizational Dynamics, 3(1), 2-18. doi:10.1016/0090-2616(74)90001-1

Combs, J. G., Crook, T. R., \& Shook, C. L. (2005). The dimensionality of organizational performance and its implications for strategic management research. D. Ketchen, Jr., \& D. D. Bergh (Coords.) Research methodology in strategy and management (pp. 259-286). Kidlington, UK: Elsevier.

Cool, K., \& Dierickx, I. (1993). Rivalry, strategic groups and firm profitability. Strategic Manangement Journal, 14(1), 47-59. doi:10.1002/smj.4250140106
Cool, K., Dierickx, I., \& Jemison, D. (1989). Business strategy, market structure and risk-return relationships: A structural approach. Strategic Manangement Journal, 10(6), 507-522.

Dias, A. T. (2004). Competição, orientação estratégica e desempenho de empresas em ambiente turbulento: Uma abordagem empírica (Dissertação de mestrado, Centro de Pós-graduação e Pesquisa em Administração da Faculdade de Ciências Econômicas, Universidade Federal de Minas Gerais).

Dias, A. T. (2009). Análise do papel das estratégias corporativas nas relações entre ambiente e desempenho (Tese de doutorado, Centro de Pós-graduação e Pesquisa em Administração da Faculdade de Ciências Econômicas, Universidade Federal de Minas Gerais).

Griffiths, W., Jensen, P. H., \& Webster, E. (2011). What creates abnormal profits? Scottish Journal of Political Economy, 58(3), 323-346. doi:10.1111/j.1467-9485.2011.00549.x

Hatten, K. J., \& Schendel, D. E. (1977). Heterogeneity within an industry: Firm conduct in the U. S. brewing industry, 1952-71. Journal of Industrial Economics, 26(2), 97-113. doi:10.2307/2097932

Hawawini, G., Subramanian, V., \& Verdin, P. (2003). Is performance driven by industry-or firm- specific factors? A new look at the evidence. Strategic Management Journal, 24(1), 1-16. doi:10.1002/smj.278

Hawawini, G., Subramanian, V., \& Verdin, P. (2005). Is performance driven by industry or firm-specific factors? A reply to McNamara, Aime, and Vaaler. Strategic Management Journal, 26(11), 1083-1086. doi:10.1002/smj.500

Keats, B., \& Hitt, M. A. (1988). A causal model of linkages among environmental dimensions, macro organizational characteristics, and performance. Academy of Management Journal, 31(3), 570-598.

Kim, H., \& Reinschmidt, K. F. (2012). Market structure and organizational performance of construction organizations. Journal of Management Engineering, 28(2), 212-220. doi:10.1061/(ASCE)ME.19435479.0000082

Lewin, A. Y., \& Minton, J. W. (1986). Determining organizational effectiveness: Another look, and an agenda for research. Management Science, 32(5), 514-538. doi:10.1287/mnsc.32.5.514

Mas-Ruiz, F., \& Ruiz-Moreno, F. (2011). Rivalry within strategic groups and consequences for performance: The firm-size effects. Strategic Management Journal, 32(12), 1286-1308. doi:10.1002/smj.936

Matitz, Q. R. S., \& Bulgacov, S. (2011). O conceito desempenho em estudos organizacionais e estratégia: Um modelo de análise multidimensional. RAC-Revista de Administração Contemporânea, 15(4), 580-607. doi:10.1590/s1415-65552011000400003

Mauri, A. J., \& Michaels, M. P. (1998). Firm and industry effects within strategic management: An empirical examination. Strategic Management Journal, 19(3), 211-219. doi:10.1002/(sici)10970266(199803)19:3〈211::aid-smj947〉3.0.c0;2-t

Murthi, B., Rasheed, A. A., \& Goll, I. (2013). An empirical analysis of strategic groups in the airline industry using latent class regressions. Managerial \& Decision Economics, 34(2), 59-73. doi:10.1002/ mde.2574

Neter J., Kutner, M. H., Nachtsheim, C. J., \& Wasserman, W. (1996). Applied linear statistical models (4th ed.). Nova York, EUA: McGraw-Hill.

Pimentel, R. C., \& Aguiar, A. B. de. (2016). The role of earnings persistence in valuation accuracy and the time horizon. RAE-Revista de Administração de Empresas, 56(1), 71-86. doi:10.1590/So034759020160107 
Porter, M. E. (1979). The structure within industries and companies' performance. The Review of Economics and Statistics, 61(2), 214-227. doi:10.2307/1924589

Porter, M. E. (1981). The contributions of industrial organization to strategic management. Academy of Management Review, 6(4), 609620. doi:10.5465/amr.1981.4285706

Porter, M. E. (2004). Estratégia competitiva: Técnicas para análise de indústrias e da concorrência ( $12^{\mathrm{a}} \mathrm{ed}$.). Rio de Janeiro, RJ: Campus.

Powell, T. C. (2001). Competitive advantage: Logical and philosophical considerations. Strategic Management Journal, 22(9), 875-888. doi:10.1002/smj.173

Prescott, J. E. (1986). Environments as moderators of the relationship between strategy and performance. Academy of Management Journal, 29(2), 329-346. doi:10.2307/256191

Richard, P. J., Devinney, T. M., Yip, G. S., \& Johnson, G. (2009). Measuring organizational performance: Towards methodological best practice. Journal of Management, 35(3), 718-804. doi:10.1177/0149206308330560

Roquebert, J. A., Phillips, R. L., \& Westfall, P. A. (1996). Markets vs. management: What 'drives' profitability? Strategic Management Journal, 17(8), 653-664. doi: 10.1002/(sici)10970266(199610)17:8<653::aid-smj840>3.0.c0;2-0

Rumelt, R. P. (1991). How much does industry matter? Strategic Management Journal, 12(3), 167-185. doi:10.1002/smj.4250120302

Santos, J. B. (2008). Uma proposta de representação e conceituação do desempenho empresarial (Dissertação de mestrado, Escola de Administração de Empresas de São Paulo da Fundação Getulio Vargas).

Schilke, 0. (2014). On the contingent value of dynamic capabilities for competitive advantage: The nonlinear moderating effect of environmental dynamism. Strategic Management Journal, 35(2), 179203. doi:10.1002/smj.2099
Schmalensee, R. (1985). Do markets differ much? American Economic Review, 75(3), 341-351.

Sharma, V. (2011). Stock returns and product market competition: Beyond industry concentration. Review of Quantitative Finance \& Accounting, 37(3), 283-299. doi:10.1007/s11156-010-0205-0

Sharp, B. M., Bergh, D. D., \& Li, M. (2013). Measuring and testing industry effects in strategic management research: An update, assessment, and demonstration. Organizational Research Methods, 16(1), 43-66. doi:10.1177/1094428112470847

Simerly, R. L., \& Li. M. (2000). Environmental dynamism, capital structure and performance: A theoretical integration and an empirical test. Strategic Management Journal, 21(1), 31-49. doi:10.1002/(sici)10970266(200001)21:1く31::aid-smj76»3.0.co;2-t

Tan, J. J., Litschert, R. J. (1994). Environment-strategy relationship and its performance implications: An empirical study of the Chinese electronics industry. Strategic Management Journal, 15(1), 1-20. doi:10.1002/smj.4250150102

Tarziján, J., \& Ramirez, C. (2010). Firm, industry and corporation effects revisited: A mixed multilevel analysis for Chilean companies. Applied Economics Letters, 18(1), 95-100. doi:10.1080/13504850903425165

Tirole, J. (1988). The theory of industrial organization. Cambridge, EUA: MIT Press.

Venkatraman, N., \& Ramanujam, V. (1986). Measurement of business performance in strategy research: A comparison of approaches. Academic of Management Review, 11(4), 801-814.

Waring, G. F. (1996). Industry differences in the persistence of firmspecific returns. The American Economic Review, 86(5), 1253-1265. 